\title{
Assessing the Status of Quality Control Parameters of Four Computed Tomography Scanners in Ibadan
}

\author{
Osayaba Peace Egharevba, Christian Chukwuemeka Nzotta, \\ Emmanuel Oyeyemi Oyekunle, and Mohammed Anas
}

\section{ABSTRACT}

Background: Quality control (QC) of computed tomography (CT) scanners is important to evaluate succinctly quality image and radiation dose obtainable in a clinical environment. The aim of this study was to evaluate the quality of images generated by $\mathrm{CT}$ scanners used at some diagnostic facilities in Ibadan, Nigeria.

Materials and Methods: A cross sectional design was employed in this study, four centers were studied, one government hospital and three private hospitals. The head CT phantom was used to verify the accomplishment of the CT scanners performance to the international quality requirements. Regions of interest were selected at the center of the image and at the periphery to obtain results for the CT number for water test, uniformity test, noise, and artifact test.

Results: The mean CT number for water across the centers ranged from $-0.12 \mathrm{HU}$ to $-2.2 \mathrm{HU}$ which were within $\pm 3 \mathrm{HU}$ recommended by the equipment manufacturer. Values of standard deviation of the mean CT number ranged from 2.41 to $5.77 \mathrm{HU}$ which to a little extent exceeded the set $\pm 5 \mathrm{HU}$ tolerance range. Similarly, the presence of streak artifact was observed in the images obtained at one center.

Conclusion: Two out of the four computed tomography scanners assessed passed the four tests performed. Noise and artifact were the problem observed at centers $B$ and $C$ respectively. There was however no likelihood of periodic performance of these basic quality control tests at two of the centers in this study. Adequate records of quality control data should be kept regularly to allow in-depth analysis of failure rates of different tests, changes occurring during equipment lifetime and comparisons among CT scanners.

Keywords: computed tomography, image quality, quality control.
Submitted : May 02, 2021

Published : July 25, 2021

ISSN: $2593-8339$

DOI: $10.24018 /$ ejmed.2021.3.4.849

\section{O. P. Egharevba*}

Department of Radiography and Radiological Sciences, College of Health Sciences, Nnamdi Azikiwe University, Nnewi Campus, Anambra state, Nigeria

(e-mail: ask4osayaba@gmail.com)

C. C. Nzotta

Department of Radiography and Radiological Sciences, College of Health Sciences, Nnamdi Azikiwe University, Nnewi Campus.

(e-mail: nzottac@yahoo.com)

E. O. Oyekunle

Department of Radiation Oncology,

University College Hospital - Ibadan,

Oyo state, Nigeria.

(e-mail:

emmanueloyekunle.eo@gmail.com)

M. Anas

Department of Radiology, State Specialist Hospital - Gombe, Gombe state, Nigeria.

(e-mail: anastinja5490@gmail.com)

*Corresponding Author

\section{INTRODUCTION}

Computed tomography (CT) is a medical $\mathrm{x}$-ray imaging modality that transmits numerous $\mathrm{x}$-ray beams through the human body to obtain images for disease diagnosis, evaluation of clinical treatment, and assessment of patient wellness [1]. This is mainly significant for the diagnosis of pathological conditions involving soft tissue. Sustainability of high standard X-ray equipment for high quality radiographs can only be achieved if comprehensive Quality control program have been implemented [2]. There are many causes of these breakdowns, some of which could be careless handling of the machines, proper care of CT is very important since the equipment is very expensive [3]. Other causes could be the lack of regular servicing of the CT equipment by trained and competent maintenance personnel and the lack of spare parts for the equipment and the absence of regular Quality Control (QC) testing of equipment. One important element of taking care of equipment is to conduct Quality Control tests.

Quality control are systematic and procedural checks, tests and measurement done to a system to ensure standardization or conformance to a known standard. However, there may be significant variability in the quality of CT performance at different sites. The American College of Radiology (ACR) has initiated a voluntary CT accreditation program. This program has followed the approach of previous ACR accreditation programs, which have established practices and standards for quality control tests some of which should be carried out weekly, monthly, and annually. The goal of any QC program is to include sufficient testing to ensure good image quality and patient safety but not to include so many tests that it becomes impractical in a clinical environment and unreasonable to the staff. CT manufacturers provide a list of scanner specific test and tolerances that should be performed on a routine basis. These vendors provided descriptions can 
be used as the basis of a structured QC program [4].

Routine QC can help ensure the equipment is operating appropriately to ensure high quality diagnostic images are produce with the least exposure to ionizing radiation. For the purpose of this paper, QC will refer to the simple daily test performed on CT scanner to monitor some basic aspect of scanner performance which include. CT number for water, CT field uniformity and CT noise and artifact. Manufacturer's specific tests are not only conducted to maintain equipment operation at an acceptable level, but these programs are also designed to recognize and create a corrective action for quality assurance issues [5].

New phantoms are constantly being developed to evaluate problem areas in maintaining quality images. Proper use of quality assurance phantoms for daily QA testing, recording daily test data, as well as understanding tests described in this module remain a duty of the CT technologist.

A good quality assurance program containing accurate documentation, along with the newest testing devices, are necessary in maintaining the highest standard of quality and the minimum quantities of radiation exposure possible to our patients. Due to the excellent diagnostic information that CT provides; it will continue to be an essential tool for medical diagnosis. The aim of this study was therefore to evaluate the image quality of 4 scanners installed at public and private centers in the ancient of Ibadan, Nigeria.

\section{MATERIALS AND METHOD}

The study was carried out at four centers, Centers A, B, C, and D all in Ibadan, Oyo state, Nigeria with the head CT water phantom of $20 \mathrm{~cm}$ in diameter used to measure image quality parameters. Using the brain protocol for the scan. CT images for each parameter were obtained and measured from a single scan. With the aid of a phantom holder, the phantom was placed on the tabletop and then aligned such that it will be at the center of the gantry in the axial, sagittal and coronal planes. The CT external or internal alignment laser lights were accurately position over the center of the phantom. At each of the centers, the head phantom was scanned using the head protocol for each center. The specifications of the CT scanners and the scanning parameters used for this study are presented in Table I. The mean CT number test was performed at the four centers with the aid of water phantom test tool. Five regions of interest (ROI) of about $2-3 \mathrm{~cm}$ or 300 pixels were measured for the average CT numbers. The ROIs were placed at the center, top, bottom, left and right of the acquired images while the mean CT numbers were measured. Image noise was deduced from the evaluated CT number for water phantom test. This is normally a function of the standard deviation within each ROI as measured by the computed tomography software.

The CT field uniformity was assessed by considering the mean water values obtained at the 5 regions of interest. Field uniformity test was considered satisfactory when mean CT numbers for the identified five ROIs were within the tolerance values of $5 \mathrm{HU}$. The images from each center were also inspected for any artifacts.

\section{RESULTS}

The mean CT number for water across the centers ranged from -0.12 to $-2.2 \mathrm{HU}$ while the standard deviation of the mean CT number ranged from 2.2 to 5.8 as presented in Table II.

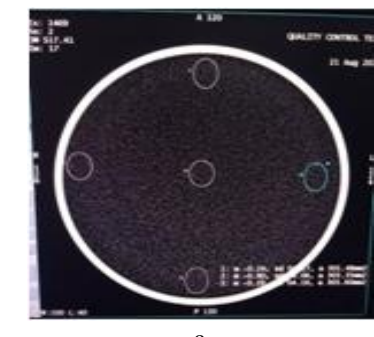

a

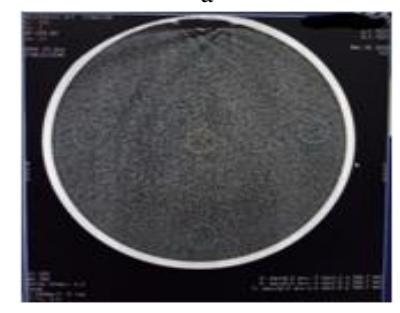

$\mathrm{c}$

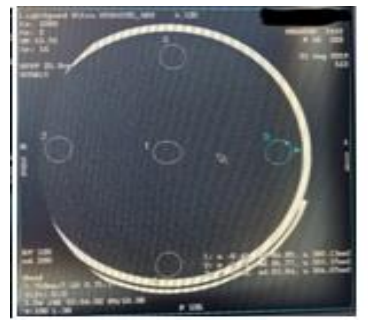

b

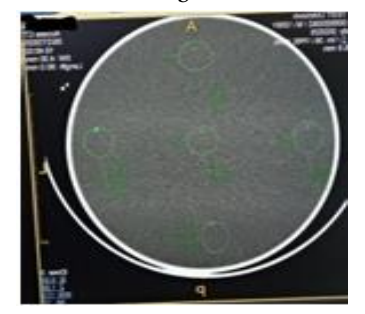

d
Fig. 1 Images of the CT number for water and Field Uniformity obtained from water Phantom scan at (a) center A and (b) center B (c) center C (d) center $\mathrm{D}$ are shown.

TABLE I: COMPUTED TOMOGRAPHY SCANNERS AT THE FOUR

\begin{tabular}{ccccccc}
\multicolumn{7}{c}{} \\
\hline & Vendor & Model & Year of Installation & $\mathrm{kVp}$ & $\mathrm{mA}$ & Slice Thickness \\
\hline A & GE & Brivo CT & 2015 & 120 & 180 & 5.00 \\
B & GE & 385 series & 2015 & 120 & 200 & 3.75 \\
C & GE & LightSpeed & 2018 & 100 & 160 & 5.00 \\
D & PHILLIPS & Brivo CT 385 series Access & 2019 & 120 & 210 & 4.00 \\
\hline
\end{tabular}

TABLE II: MEAN CT NUMBERS FOR WATER AND STANDARD DEVIATION FOR 5 REGIONS OF INTEREST AT THE FOUR STUDY CENTERS IN IBADAN, NigERIA

\begin{tabular}{ccccccccc}
\hline & \multicolumn{9}{c}{ Centers } \\
\cline { 2 - 9 } ROI & \multicolumn{2}{c}{ A } & \multicolumn{1}{c}{ B } & \multicolumn{2}{c}{ C } \\
\cline { 2 - 9 } 1 & Mean & Std. & Mean & Std. & Mean & Std & Mean & Std. \\
2 & -0.24 & 4.17 & -0.41 & 4.89 & -0.40 & 3.82 & -1.60 & 2.50 \\
3 & -0.90 & 4.06 & -1.62 & 5.77 & -0.72 & 3.01 & -2.21 & 2.60 \\
4 & -0.29 & 4.19 & -0.12 & 3.84 & -0.51 & 3.20 & 0.31 & 2.80 \\
5 & -0.33 & 5.01 & -0.97 & 4.87 & -0.40 & 3.00 & 0.50 & 2.41 \\
Uniformity & -1.19 & 4.22 & -1.05 & 4.55 & -1.40 & 3.90 & 0.20 & 2.42 \\
Noise & Passed & \multicolumn{2}{c}{ Passed } & Passed & Passed \\
Artifact & Passed & \multicolumn{2}{c}{ Failed } & Passed & Passed \\
\hline
\end{tabular}




\section{DISCUSSION}

Quality control testing of Computed Tomography scanners should ideally include a measurement of CT numbers. The CT number values are clinically relevant in determining the composition of various tissues in the body and accuracy is important in the characterization of tumors. Effective QC requires that tolerance ranges of $\mathrm{CT}$ number values be defined and a measured value outside the range indicates the need for further investigation and possible recalibration of the scanner [6].

Evaluation of CT number for water and field uniformity test carried out was done to check the reconstruction algorithm that computes CT numbers across the images. A region of interest on an image from a scanner is said to pass noise test if the standard deviation related to the CT number is $\leq 5 \mathrm{HU}$. At center A, the scanner's values were within the tolerance values stipulated by the manufacturer for the mean CT water values, noise, and uniformity. Figure (1a) also suggest that the test for artifact was also passed. These satisfactory parameters imply that good quality images at the least radiation dose are expected from this center. The positive outcomes of the test could be attributed to periodic presence of a visiting medical physicist who conducted quality control test on a regular basis at the center.

At center B, the CT scanner at the center yielded mean water values within tolerance limits and as such passed CT number for water and uniformity tests. Values of standard deviation obtained at the peripheral ROIs were 5.77, 3.84, 4.87, and 4.55. HU. This indicates a failure in the noise test carried out because the value exceeds the manufacturer's specification of $\pm 5 \mathrm{HU}$.

The possible cause of noise in CT images is number of detected photons, matrix size, slice thickness, algorithm, electronic noise, and scattered radiation. The implication of this is that low contrast resolution will be limited, and it may hide anatomy similar to surrounding tissue in images and can lead to misinterpretation of result. This might lead to wrong diagnosis as agreed with the study conducted by Sidi [7] on evaluation of some quality control parameters in diagnostic computed tomography scanners in Kano metropolis, Nigeria.

The findings at center $\mathrm{C}$ were largely similar to that of center A given that CT number, uniformity and noise tests were passed. However, the images (Fig. 1a and 1c) obtained at center $\mathrm{C}$ indicated acceptable quality as well as a type of image artifacts (streak artifact). It is in agreement with the study conducted by Mansour et. al., [8] on quality control of CT image using American College of Radiology phantom. Streak artifact occurs in images due to the presence or movement of objects of very high density, like metallic clip, metallic implants, and contrast media. They are seen as radiating dense white streak as seen in Fig. 1c. Streak artifact can be reduced using newer reconstruction technique or metal artifact reduction software. The result of the uniformity and high contrast resolution test is in agreement with the work of Nookala [8] on modification of CT quality assurance phantom for PET/CT alignment and PET resolution.

The outcomes of quality control checks at center D as shown in Table II and Fig. $1 \mathrm{~b}$ and $1 \mathrm{~d}$ are considerably similar to that of center A, and the existing CT scanner was therefore considered to have passed the four tests performed. The presence of a medical physicist who is resident at the center could have therefore contributed to the positive outcomes of the test performed. The manufacturer of the scanner in this case (Phillips Healthcare) had recommended zero value as water CT number but it is permitted to be within $\pm 4 \mathrm{HU}$ at the center of the image and $\pm 5 \mathrm{HU}$ at the periphery. This is in agreement with a previous work done in Urmia, Iran [9]. The result also disagrees with the work of Nute et al. [4], which had a high failure rate, for noise, artifact, and uniformity. A previous study carried out was done in Kano state, by Sidi [7] who recommended that performance of these basic quality control tests for CT must be done at other centers across the country.

The outcomes of this study showed that 2 of the 4 evaluated CT scanners had passed the four basic quality control tests which would assure quality images at the least radiation dose. The tests considered in this study are fundamental as these would determine whether or not diagnostic image quality would be achieved using minimal radiation dose at the study centers. Further studies however should be carried out to include all quality control tests for computed tomography scanners as prescribed by the American College of Radiology.

Although outcomes of basic performance tests conducted on the equipment at the four locations were largely satisfactory, the study revealed a lack of periodic quality assurance tasks. This study has strongly indicated the need for timely implementation of quality assurance program and corrective action should be initiated by biomedical engineers on CT scanners that fail required tests. Adequate records of quality control data including images should be kept regularly to allow in-depth analysis of failure rates of different tests, changes occurring during equipment lifetime and comparisons among CT scanners.

\section{ACKNOWLEDGMENT}

The authors would like to acknowledge the management of the concerned hospitals for their approval to undertake this study as well as the radiographers for their cooperation and Mr. Olubunmi Ogunmayowa for his funding during the course of this study.

\section{REFERENCES}

[1] E. Seeram, (2001), Computed tomography technology, Philadelphia, WB Saunders.

[2] P. Lloyd, (2001), Quality Assurance Workbook for Radiographers \& Radiological Technologists. World Health Organization, Geneva.

[3] E. Burns, (1992), Radiographic Imaging. 2ndEd. Saunders, USA.

[4] L. Nute, R. John, M. Donna, J. Brandon, D. Dianna, (2013), Evaluation of over 100 years of computed tomography daily quality control data. Available at: http://www.ncbi.nlm.nih.gov/pubmed/23635278.

[5] J. Hsieh, (2003), Investigation of an image artifact induced by projection of noise in homogeneity in multislice helical computed tomography. Physmed, pp. 345-356.

[6] M. Sidi, (2016), Evaluation of some quality control parameters in Diagnostic computed tomography scanners in Kano metropolis, Nigeria. Indiana Journal of Applied Research, vol. 6, Issue 3. pp. 205207.

[7] Z. Mansour, A. Mokhtar, A. Sarhan, M. Ahmed, T. El-Diasty, (2016), Quality Control of CT image using American College of Radiology (ACR) phantom, The Egyptian Journal of Radiology and Nuclear Medicine, vol. 47, issue 4, December 2016, pp. 1665-1671. 
[8] P. Nookala, (2005), Modification of CT assurance phantom for PET/CT alignment and PET resolution. LSU Master's Thesis. 4202.

[9] G. Bagher, E. Mahnaz, B. Zhaleh, Z. Reza, R. Soheila, (2018), Assessment of water CT number, field uniformity and noise in diagnostics computed tomography scanners in Urmia metropolis, Iran. International Journal of Advanced Biotechnology and Research, vol. Issue 1:0976-2612. Available at: http://www.bipublication.com.

[10] R. J. Cropp, P. Seslija, D. Tso, Y. Thakur, Scanner and kVp dependence of measured CT numbers in the ACR CT phantom. J Appl Clin Med Phys., 2013; 14(6): 4417. doi:10.1120/jacmp.v14i6.4417. 